\title{
Knee osteoarthritis in a chestnut farmer - Case Report
}

\author{
Stefano Mattiolii', Francesca Graziosi', Stefania Curti ${ }^{1}$, Roberta Bonfiglioli', Antonio Argentino ${ }^{1,2}$, \\ Francesco Saverio Violante ${ }^{1}$ \\ ${ }^{1}$ Department of Medical and Surgical Sciences, University of Bologna, Italy \\ ${ }^{2}$ School of Occupational Medicine, Italy
}

Mattioli S, Graziosi F, Curti S, Bonfiglioli R, Argentino A, Violante F.S. Knee osteoarthritis in a chestnut farmer - Case Report. Ann Agric Environ Med. 2017; 24(1): 148-150. doi: 10.5604/12321966.1233903

\begin{abstract}
Introduction. Several studies have dealt with the issue of professional risk factors and onset of knee osteoarthritis (OA). In particular, occupational epidemiological studies have provided evidence that activities resulting in biomechanical overload may be linked with an increased risk of knee OA - also among farmers. To our knowledge, no cases of knee OA among chestnut farmers have been reported in the literature.

Case report. We report the case of a 70-year-old Caucasian male who has worked for more than 50 years on a chestnut farm. In 2007, an X-ray and a MRI, performed after a workplace accident to his left knee, showed the presence of knee OA. His job required a range of repetitive tasks, such as squatting, kneeling, climbing, walking on sloping terrain, assuming uncomfortable postures, and lifting and carrying heavy loads for the great majority of the working day. All the aforementioned tasks are known occupational risk factors for knee OA. Regarding individual risk factors, at the time of the first diagnosis of knee OA, the worker was 64-years-old with a body mass index of $26.5 \mathrm{~kg} / \mathrm{m}^{2}$. He reported no cases of arthritis among his relatives and no sports playing on his part. In addition, his medical history revealed the presence of two minor lumbar disc herniations and tendinitis of the long head of the biceps.

Conclusion. Considering the lack of major individual risk factors for knee OA, it is reasonable to suppose that five decades of exposure to biomechanical overload as a chestnut farmer was a relevant risk factor for the onset of the disease.
\end{abstract}

Key words

knee osteoarthritis, chestnut farmer, agricultural workers' diseases, occupational exposure, biomechanical overload

\section{INTRODUCTION}

Osteoarthritis (OA) is a painful chronic joint disease that can induce some changes to the joints, such as articular cartilage loss, synovial inflammation, subchondral bone and meniscal damage, muscle weakness, ligamentous laxity, and osteophyte development. When this disease affects the knees (i.e. gonarthrosis or OA of the knee), the most commonly affected joint compartments are the medial tibiofemoral and the patellofemoral [1]. Knee OA can cause significant disability and impaired quality of life, and its development can have an impact on patients' capacity to undertake daily activities, including their ability to work [2].

With regard to individual risk factors, women and older people have an increased risk of developing this pathology. There is also evidence for the existence of ethnic differences, with higher rates in black and Chinese people than in white people [1]. Obesity and joint injury - in particular anterior cruciate ligament and meniscal injuries in people who play sports - are other important and modifiable risk factors; obesity and knee malalignment (varus for medial knee and valgus for lateral knee osteoarthritis) can promote progression of the pathology [1].

As regards occupational risk factors, there is reasonably good evidence that occupational activities involving squatting and kneeling for substantial parts of the working day, regular heavy lifting, climbing, and high physical workload are likely

Address for correspondence: Antonio Argentino, Department of Medical and Surgical Sciences, University of Bologna, Italy

E-mail:antonio.argentino@studio.unibo.it

Received: 29 July 2015; accepted: 26 September 2016; first published on January 2017 to contribute to the onset or aggravation of knee OA [2]. In particular, several studies showed an increased risk of developing knee OA among farm workers $[3,4,5,6]$.

We report a case of knee OA in a chestnut farmer along with the biomechanical risk analysis of the tasks performed in this occupation.

\section{CASE REPORT}

We present the case of a 70-year-old Caucasian male who has worked for more than 50 years on a chestnut (Castanea sativa) farm, located in the Tuscan-Emilian Apennines on a sloping terrain with an area of 10 hectares, 1,350 trees and a production per year of about 15 tons.

In 2007, the farmer had a work accident in his left knee while he was descending from a ladder. A month later, he reported to the emergency department where an X-ray showed the presence of knee OA. At that time, his body mass index was $26.5 \mathrm{~kg} / \mathrm{m}^{2}$.

Two months later, a new exacerbation of the left knee pain led him to return to the emergency department where a Magnetic Resonance Imagining (MRI) performed in his left knee excluded acute lesions, but showed severe knee OA. On the basis of this clinical situation, an orthopedic surgeon indicated the need for arthroscopic lavage with lateral release, also suggesting the need to undergo arthroplasty. At the end of 2007, the arthroscopic lateral release of the left knee was performed. In 2009, an X-ray showed signs of bilateral knee OA. Figures 1-3 show the X-ray of his left knee performed in 2011. At the beginning of 2014, he underwent arthroplasty 
of the left knee. In early 2010, the patient was examined in our operative unit. His medical history revealed the presence of other musculoskeletal disorders due to biomechanical overload: lumbar disc disease (two minor lumbar herniation in L4-L5 and L5-S1 with some lumbosacral bulging, highlighted by an MRI in 2010) and tendonitis of the long head of the biceps in his left shoulder (shown by a medical ultrasonography in 2008).

Assessment of potential biomechanical risk factors. The job evaluation included an interview to determine the worker's tasks and their frequency and duration. Observations were performed for each task in order to identify any physical stresses. Of note, different specific duties are required in each season.

In winter the chestnut farmer prunes and chops the tree branches using a $4 \mathrm{~kg}$ chainsaw for more than 8 hours a day. Then, he manually removes the branches and clears the fern undergrowth with a shoulder strap blower (10 kg weight), which he uses also for nearly 8 hours a day. He prunes 10 trees per day with an average of about 45-60 minutes per tree. The most frequent tasks performed in this period (i.e. November - March) are those related to climbing ladders for a part of the day, with the back bent forward $\left(30^{\circ}\right)$. Hand-wrist flexion/extension movements, gripping and working with the elbows raised above the shoulders, are also carried out.

In spring (specifically in April), the main task is manual plant grafting, which involves climbing ladders and holding heavy loads, such as a chainsaw for part of the working day. During this period, the tasks require frequent hand gripping and working with the elbows raised above the shoulders. The number of manual plant grafts is about 40-50 per day; every graft requires not more than 15 minutes.

In summer (May - September), he clears undergrowth; this job requires standing, walking and leaning on a sloping terrain, frequent trunk rotation, holding heavy loads, hand gripping and hand-wrist flexion-extension.

In autumn, the most important and frequent task is hand-harvesting by ground pick-up. This activity includes transporting bags of the collected chestnuts to storage, walking up-and-down a sloping terrain for about 500-600 metres. In particular, this job is performed in October for 8-10 hours a day. Approximately 4-6 bags are to be filled and carried daily, corresponding to a total of 130-150 kg handled per day. Kneeling, squatting and back bent forward $\left(30^{\circ}\right)$, together with upper limb repetitive movements (especially hand-wrist flexion-extension, hand gripping and pinching), are performed for a large part of the working day. Of note, the job also requires lifting and carrying heavy loads on a sloping terrain.

\section{DISCUSSION}

The patient's occupation may have played a role in the development of knee OA, since during 5 decades of employment as a chestnut farmer, he performed multiple tasks that exposed him to biomechanical overload of the knees.

Briefly, a chestnut farmer's job requires a range of repetitive tasks, such as squatting, kneeling, climbing ladders, walking on a sloping terrain, assuming uncomfortable postures, and lifting and carrying heavy loads for the great majority of the working day.

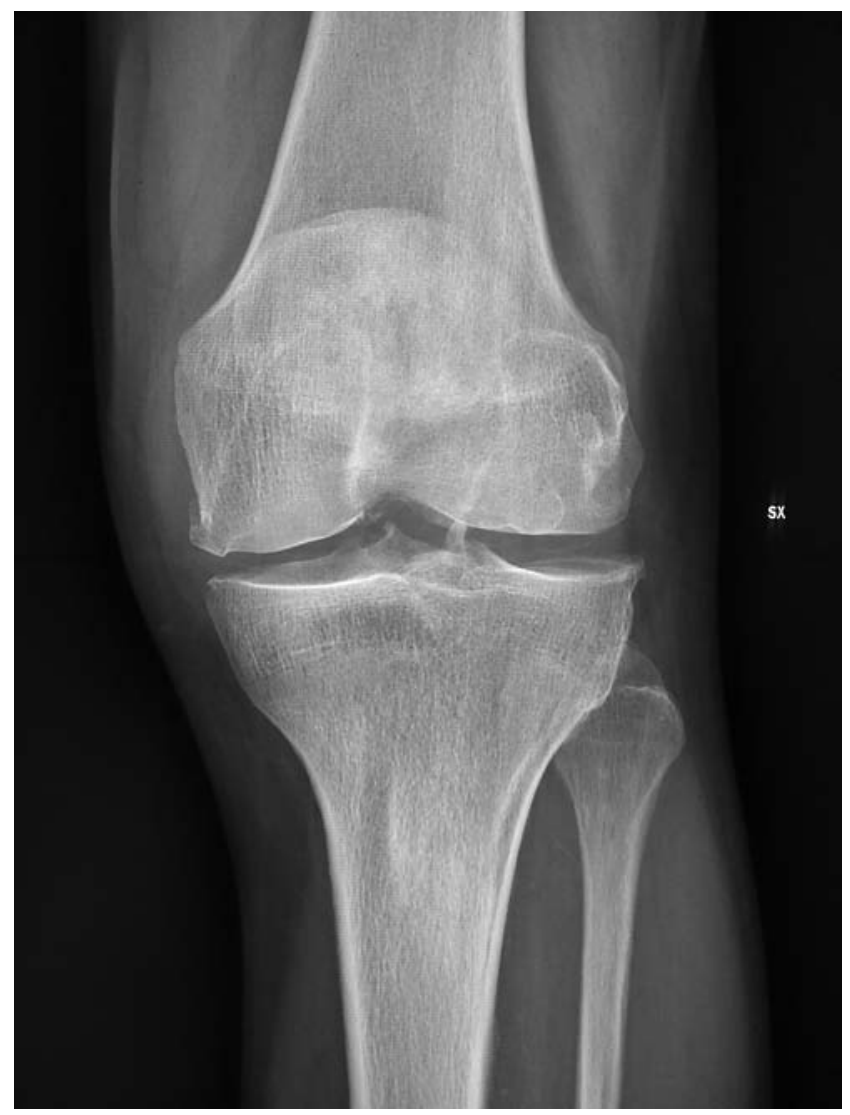

Figure 1. 2011 Left knee X-ray, anterior-posterior projection: severe tibiofemoral knee OA: joint space narrowing with osteophytes, in particular in the medial compartment

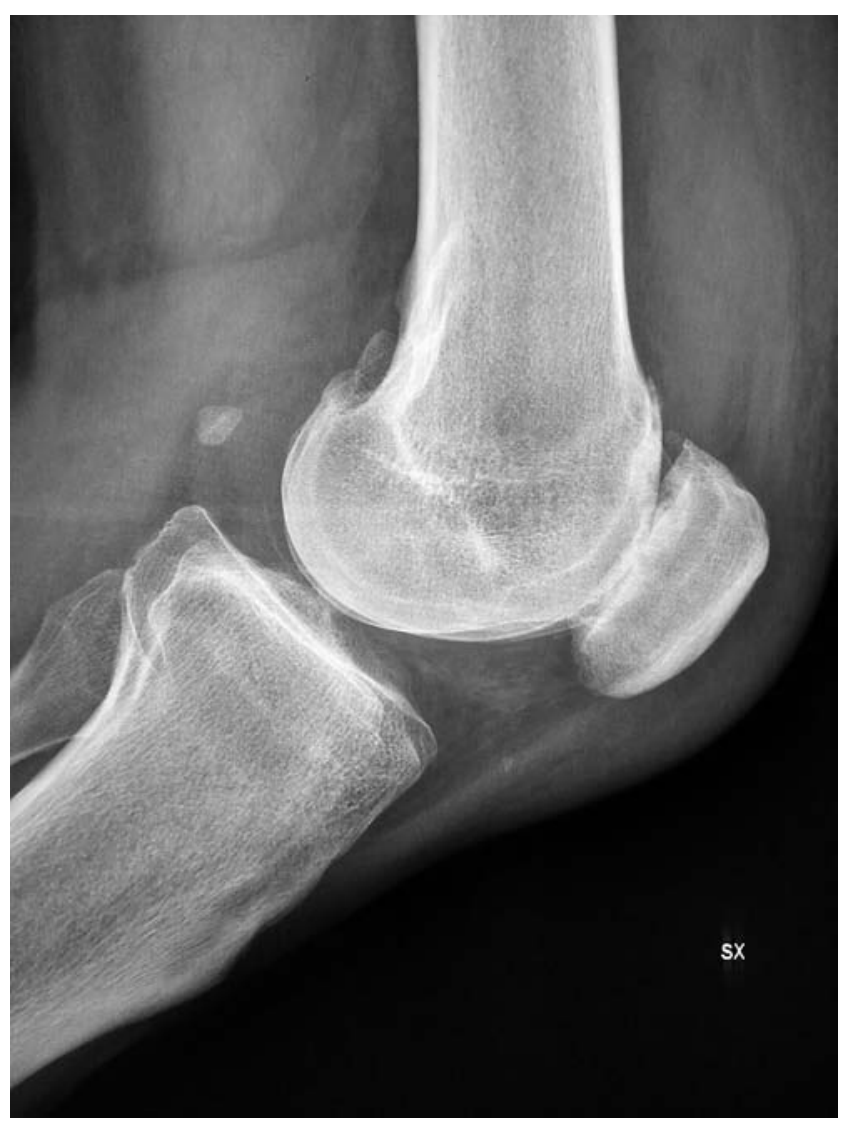

Figure 2. 2011 Left knee X-ray, lateral projection: severe patellofemoral knee OA: joint space narrowing and lateralisation of the patella 


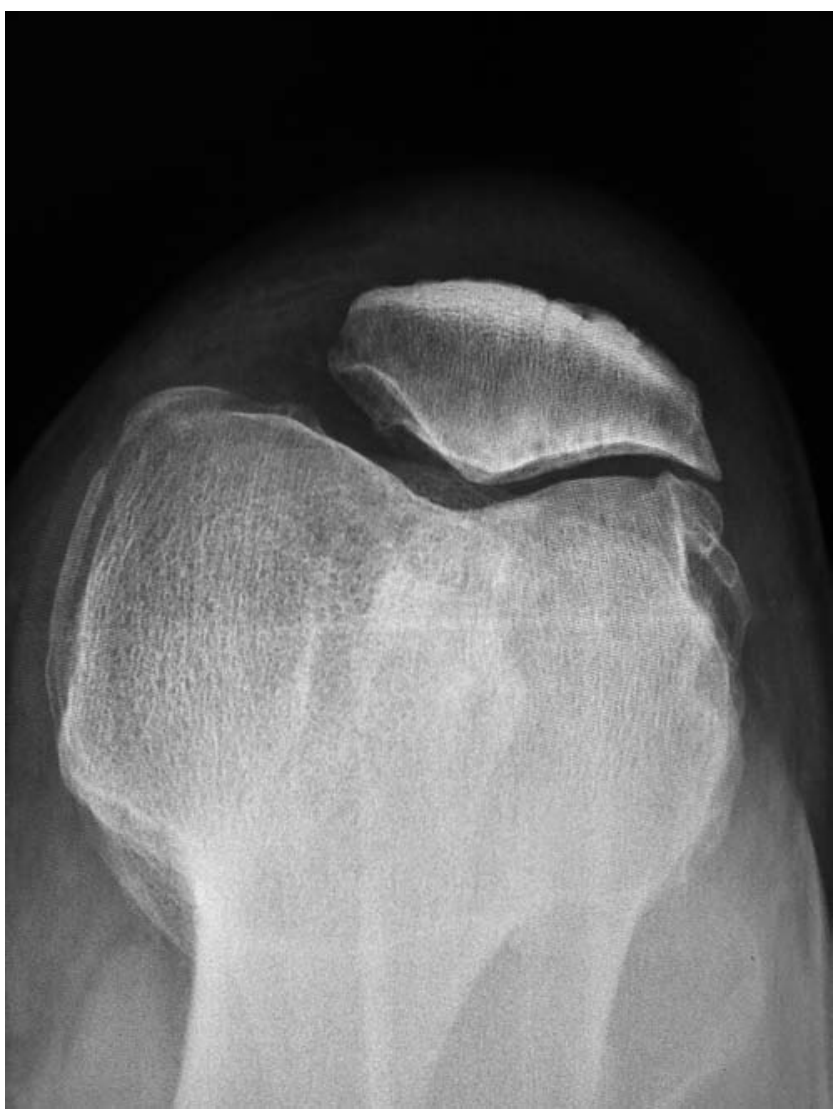

Figure 3. 2011 Left knee X-ray, axial projection: lateralisation of the patella

All the aforementioned tasks are well-known occupational risk factors, as reported by Palmer in a systematic review regarding the contribution of occupational activities in the development of knee OA [2]. Palmer concluded that there is reasonably good evidence to connect work activity and knee $\mathrm{OA}$ and - more specifically - that this evidence is stronger with regard to squatting / kneeling, lifting and physical workload, somewhat weaker for climbing and somewhat against an important effect caused by standing or walking [2]. McWilliams et al. conducted a meta-analysis on the same topic, highlighting that occupational activities such as kneeling, squatting, lifting/carrying, climbing stairs and knee bending/straining, gave positive risks for knee OA [7]. In contrast, the analyses also pointed out that standing work was not a risk factors for knee OA.

Regarding the presented case study, it should be underlined that individual risk factors (such as age and excess weight) may have also contributed, at least in part, to the onset of this pathology: diagnosis of knee OA was performed for the first time in 2007, when the worker was 64-years-old and was slightly overweight according to the World Health Organization (WHO) cut-off point of $25 \mathrm{~kg} / \mathrm{m}^{2}$ [8]. No family history of OA or sport-related injuries resulted from his personal background.

\section{CONCLUSIONS}

To our knowledge, no other cases of knee OA among chestnut farmers were previously reported in the literature.
Occupational epidemiological studies provide evidence that activities such as squatting, kneeling, climbing, lifting and physical workload may be linked with an increased risk of knee OA. All the aforementioned activities were performed by our patient during his long-lasting working life as a chestnut farmer.

Considering these aspects, together with the lack of major individual risk factors for knee OA, it is reasonable to suppose that chestnut farm work could be considered as a relevant risk factor for the onset of the disease.

\section{CONSENT}

Written informed consent was obtained from the patient for publication of this Case Report. A copy of the written consent is available for review by the Editor of this journal.

\section{List of abbreviations}

MRI: Magnetic Resonance Imagining

OA: Osteoarthritis

\section{Competing interests}

The authors declare that they have no competing interests.

\section{Authors' contributions}

SM collected the clinical data and formulated the etiological hypothesis. SM drafted the manuscript together with SC and AA. FG performed the exposure assessment and collected the exposure data. All authors participated in the interpretation of the data. FG, RB and FSV performed critical revision of the manuscript. All authors read and approved the final manuscript.

\section{Acknowledgments}

The authors express their thanks to their radiologist colleague, Dr Valentina Arrighi, for her invaluable support.

\section{REFERENCES}

1. Bennell KL, Hunter DJ, Hinman RS. Management of osteoarthritis of the knee. BMJ. 2012; 345:e4934.

2. Palmer KT. Occupational activities and osteoarthritis of the knee. Br Med Bull. 2012;102:147-70.

3. Walker-Bone K, Palmer KT. Musculoskeletal disorders in farmers and farm workers. Occup Med (Lond). 2002; 52(8):441-50.

4. Kirkhorn S, Greenlee RT, Reeser JC. The epidemiology of agriculturerelated osteoarthritis and its impact on occupational disability. WMJ. 2003; 102(7):38-44.

5. Franklin J, Ingvarsson T, Englund M, Lohmander S. Association between occupation and knee and hip replacement due to osteoarthritis: a case-control study. Arthritis Res Ther. 2010; 12(3):R102.

6. Mattioli S, Gori D, Di Gregori V, Ricotta L, Baldasseroni A, Farioli A et al. PubMed search strings for the study of agricultural workers' diseases. Am J Ind Med. 2013; 56(12):1473-81.

7. McWilliams DF, Leeb BF, Muthuri SG, Doherty M, Zhang W. Occupational risk factors for osteoarthritis of the knee: a meta-analysis. Osteoarthritis Cartilage. 2011; 19(7):829-39.

8. World Health Organization (WHO). Global Database on Body Mass Index: BMI classification. http://apps.who.int/bmi/index. jsp?introPage=intro_3.html. Accessed 28 April 2015. 